\title{
Optimization of small RNA library preparation protocol from human urinary exosomes
}

\author{
Dolores Olivares ${ }^{1 \dagger}$, Javier Perez-Hernandez ${ }^{1,2 \dagger}$, Daniel Perez-Gil ${ }^{1,3}$, Felipe J. Chaves ${ }^{4,5^{*}}$, Josep Redon ${ }^{1,6}$ \\ and Raquel Cortes $^{1 *}$ (D)
}

\begin{abstract}
Background: Sequencing of miRNAs isolated from exosomes has great potential to identify novel disease biomarkers, but exosomes have low amount of RNA, hindering adequate analysis and quantification. Here, we have assessed several steps in developing an optimized small RNA (sRNA) library preparation protocol for next-generation sequencing (NGS) miRNA analysis from urinary exosomes.
\end{abstract}

Methods: A total of 24 urinary exosome samples from donors were included in this study. RNA was extracted by column-based methods. The quality of extracted RNA was assessed by spectrophotometric quantification and Bioanalyzer software analysis. All libraries were prepared using the CleanTag small RNA library preparation protocol and the effect of our additional modifications on adapter-dimer presence, sequencing data and tagged small RNA library population was also analyzed.

Results: Our results show that good quality sequencing libraries can be prepared following our optimized small RNA library preparation protocol from urinary exosomes. When the size selection by gel purification step was included within the workflow, adapter-dimer was totally removed from cDNA libraries. Furthermore, the inclusion of this modification step within small RNA library protocol augmented the small RNA mapped reads, with an especially significant $37 \%$ increase in miRNA reads, and the gel purification step made no difference to the tagged miRNA population.

Conclusions: This study provides researchers with an optimized small RNA library preparation workflow for next generation sequencing based exosome-associated miRNA analysis that yields a high amount of miRNA mapped reads without skewing the tagged miRNA population significantly.

Keywords: Exosome, microRNA, Urine, Adapter-dimer, Size selection step, Next generation sequencing

*Correspondence: felipe.chaves@uv.es; Raquel.cortes@uv.es ${ }^{\dagger}$ Dolores Olivares and Javier Perez-Hernandez contributed equally to this work

${ }^{1}$ Cardiometabolic and Renal Risk Research Group, INCLIVA Biomedical Research Institute, Avd. Menéndez Pelayo, accesorio 4, 46010 Valencia, Spain

${ }^{4}$ Genomics and Diabetes Unit, INCLIVA Biomedical Research Institute, Avd. Menéndez Pelayo, accesorio 4, 46010 Valencia, Spain

Full list of author information is available at the end of the article

\section{Background}

Over recent years changes in circulating microRNAs (miRNAs) levels have been associated with a broad range of pathological processes. Their analysis offers various advantages which make them a potential goldmine in identification of novel biomarkers: (1) they can be found in non- or minimally invasive specimens; (2) they are relatively stable in clinical samples as regards RNase digestion, temperature variation and multiple freezethaw cycles; (3) they are involved in pathway regulation,

c) The Author(s) 2020. This article is licensed under a Creative Commons Attribution 4.0 International License, which permits use, sharing, adaptation, distribution and reproduction in any medium or format, as long as you give appropriate credit to the original author(s) and the source, provide a link to the Creative Commons licence, and indicate if changes were made. The images or other third party material in this article are included in the article's Creative Commons licence, unless indicated otherwise in a credit line to the material. If material is not included in the article's Creative Commons licence and your intended use is not permitted by statutory regulation or exceeds the permitted use, you will need to obtain permission directly from the copyright holder. To view a copy of this licence, visit http://creativeco mmons.org/licenses/by/4.0/. The Creative Commons Public Domain Dedication waiver (http://creativecommons.org/publicdomain/ zero/1.0/) applies to the data made available in this article, unless otherwise stated in a credit line to the data. 
showing tissue and cell-specific expression profiles [1]. Screening for miRNA signatures associated with different pathologies has an important role in clinical research [2-4]. The last few years have therefore seen increasing development and optimization of the various miRNA purification, detection and analysis protocols, of which next generation sequencing (NGS) is a powerful tool to detect RNA molecules in biological samples [5-7].

Our group has previously reported an association between exosomal miRNAs and albuminuria in hypertension, and renal damage in systemic lupus erythematosus $[8,9]$. Exosomes, 40-130 nm membrane-derived vesicles, have been identified as novel carriers for intercellular genetic material exchange and communication $[10,11]$, containing various nucleic acid species including mRNAs, small non-coding RNAs (sncRNAs) and particularly miRNAs $[12,13]$. Consequently, there is growing interest in their use as non-invasive biomarkers for disease diagnosis and for monitoring disease recurrence, overall comprehensive analysis of the entire miRNA repertoire of exosomes in important diseases such as cancer, immune disorders and cardiovascular disease [14-16].

However, there is a limiting factor, the lower amount of small RNA (sRNA) present in exosomes than in tissues, cell cultures or biofluids like plasma makes it difficult to obtain good quality sRNA libraries for NGS analysis. Thus, previous studies presented sRNA library protocols modified to low RNA template input to avoid adapterdimer formation, for example with an extra adapter dilution, using chemical modified adapters or with an additional gel purification step $[17,18]$, but not with RNA from exosome samples. Accordingly, there is interest in developing an optimized protocol of small RNA libraries to avoid this problem.

The objective of this study is to provide researchers with an improved sRNA library preparation workflow for NGS analysis of miRNAs from urinary exosomes. We tested the effect of low RNA input and an additional purification step on adapter-dimer formation, sRNA mapped reads and tagged library population.

\section{Methodology \\ Samples}

A total of 24 samples of urinary exosomes from donor patients were analyzed and raw data are included in the BioProject PRJNA590749, (Additional file 1: Table S1). All samples were processed in duplicate, one without size exclusion of PCR products and other using Polyacrylamide Gel Electrophoresis (PAGE) gel purification for size exclusion of PCR products. Fresh, first morning urine samples $(50 \mathrm{~mL})$ were collected in sterile containers and processed within $1 \mathrm{~h}$ after collection. Written informed consent was obtained from all donors and the study was approved by the Ethics Committee of the Hospital Clínico Universitario of Valencia and performed according to the Declaration of Helsinki.

\section{Isolation of urinary exosomes}

Exosomes were isolated from urine specimens using a combination of centrifugation, ultracentrifugation, and DTT treatment, as previously described [8]. In brief, urinary cells and debris were removed by centrifugation at $2250 \mathrm{~g}$ for $30 \mathrm{~min}$ at $4{ }^{\circ} \mathrm{C}$. Next, $50 \mathrm{~mL}$ of the collected supernatant were transferred to clean tubes with $4.2 \mathrm{~mL}$ of protease inhibitor cocktail (Sigma, Missouri, USA) and centrifuged at $20,000 \mathrm{~g}$ for $45 \mathrm{~min}$ at $4{ }^{\circ} \mathrm{C}$ to eliminate large microvesicles (Ultracentrifuge Optima L $100 \mathrm{~K}, 70$ Ti rotor, Beckman Instruments, CA, USA). The supernatant was spun in an ultracentrifuge at $121,000 \mathrm{~g}$ for $70 \mathrm{~min}$ at $4{ }^{\circ} \mathrm{C}$, obtaining exosome-depleted supernatant. Exosome pellets were treated with DTT to eliminate protein complexes, washed with sterile RNase-free PBS and ultracentrifuged again at $121,000 \mathrm{~g}$ for $70 \mathrm{~min}$ (Ultracentrifuge Optima L $100 \mathrm{~K}, 70.1$ Ti rotor, Beckman Instruments, CA, USA). Exosome pellets from $50 \mathrm{~mL}$ urine were suspended in $100 \mu \mathrm{L}$ of sterile RNase-free PBS and immediately processed to extract RNA, as described below.

\section{RNA extraction}

Total RNA was extracted from exosome pellets in $100 \mu \mathrm{L}$ of exosome suspension using a Total exosome RNA and protein isolation kit (Invitrogen, Life Technologies, CA, USA) according to the manufacturer's instructions, and stored at $-80{ }^{\circ} \mathrm{C}$. Total RNA was quantified with NanoDrop ND-1000 spectrophotometer (Thermo Fisher Scientific, Waltham, MA, USA), and 2100 Bioanalyzer (Agilent ${ }^{\circledR}$ Technologies, Inc., Santa Clara, CA, USA). A RNA 6000 Pico chip run was performed afterwards for analysis and quantification of RNA eluates. The extracted RNA was stored at $-80^{\circ} \mathrm{C}$ until further analysis.

\section{Small RNA sequencing}

sRNA transcripts were converted into barcoded cDNA libraries. Library preparation was performed with CleanTag Small RNA library preparation (TriLink Biotechnologies, San Diego, USA) followed by sRNA-Seq on the Illumina HiSeq 2000 platform (CNAG, Barcelona, Spain). This sRNA library kit contains chemically modified adapters and reagents to convert sRNA to corresponding cDNA libraries for NGS, suppressing adapter-dimer formation, which is optimized for low total RNA template input [17]. Limited RNA quantity from urinary exosome specimens led to library preparation following $10 \mathrm{ng}$ total RNA template input. Multiplex adaptor ligations, reverse transcription primer hybridization, reverse transcription 
reaction and the PCR amplification were processed following library preparation protocol (Protocol \# L-3206, TriLink Biotechnologies, San Diego, USA) according to the manufacturer's instructions. When working with lower RNA input, the protocol offers modifications at several steps, for example a 1:4 adapter dilution in the adapter ligation step and 18 cycles for PCR amplification. These modifications, together with the use of chemical CleanTag modified adapters, are designed to improve ligation efficiency and eliminate adapter-dimer formation. We used the Index Primer Set 1 (Primers 1-12 with RT) and Index Primer Set 2 (Primers 13-24) from Illumina ${ }^{\circledR}$ (Illumina, San Diego, CA, USA).

After PCR pre-amplification, the cDNA constructs were loaded onto the ABI 3730 (Applied Biosystems, CA, USA) for DNA fragment analysis by capillary electrophoresis according to manufacturer's protocol. This method measures the relative size of DNA fragments with very high resolution and reproducibility, by capillary electrophoresis of fluorescent labelled DNA fragments, using internal fluorescent size standards $\left(G^{2} n e S c a n^{\mathrm{TM}} 500 \mathrm{LIZ}^{\mathrm{TM}}\right.$ dye Size Standard, ThermoFisher, Massachusetts, USA).

Electropherograms obtained were analyzed with GeneMapper software 5.0 (Applied Biosystems, CA, USA). The size selection of amplified cDNA libraries are described in the section below. The cDNA libraries were qPCR-quantified using a KAPA library quantification kit by LightCycler 480 II (Roche, Basilea, Germany). A final concentration of $20 \mathrm{nM}$ per library was used to generate the two pools, with or without the size selection step (24 samples per pool). Experiments were designed containing 24 samples which would be barcoded, pooled equally, and then loaded onto one lane of a flow cell. Libraries were prepared individually and barcoded with reverse primers during the PCR step which contained Illumina-compatible indices \#1-24. The sequence libraries obtained were subjected to the Illumina sequencing pipeline, passing through clonal cluster generation on a single-read flow cell by bridge amplification on the cBot (TruSeq SR Cluster Kit v3-cBOT-HS, Illumina, San Diego, CA, USA) and 50 cycles were sequenced by synthesis on the HiSeq 2000 (Illumina, San Diego, CA, USA).

\section{Size selection of amplified cDNA libraries}

To test whether size selection was a necessary step to obtain higher quality sequencing data, $5 \mu \mathrm{L}$ of PCR products together with $5 \mu \mathrm{L}$ TBE-Urea Sample Buffer were loaded onto TBE-Urea gel (15\%) in a vertical electrophoresis chamber, XCell Sure Lock Mini Cell (Invitrogen, Carlsbad, CA, United States), at $180 \mathrm{~V}$ and $13 \mathrm{~mA}$ for $4 \mathrm{~h}$ at room temperature. Next, gels were stained at $1 \times$ dilution for $20 \mathrm{~min}$ at dark (GelStar nucleic acid gel stain, Lonza, Basilea, Switzerland) and washed three times afterwards. To ensure the correct size of extracted bands, we developed and included in the gel three amplicons with different known sizes as markers $(120 \mathrm{pb}, 140 \mathrm{pb}$ and $150 \mathrm{pb})$ corresponding to adapter-dimer, miRNAs and other sRNA species, respectively. Bands of samples, approximately $138 \mathrm{bp}$ to $152 \mathrm{bp}$ in size were cut out and passed on to gel extraction with $100 \mu \mathrm{L}$ of Tris-EDTA in individual eppendorfs, then stored for $30 \mathrm{~min}$ at $-80{ }^{\circ} \mathrm{C}$ until frozen solid. Next, they were quickly thawed for $5 \mathrm{~min}$ at $95{ }^{\circ} \mathrm{C}$ in a thermal block, to ensure optimal recovery to PCR products. This freeze-rapid thaw approach greatly increases yield by allowing ice crystals to break apart the acrylamide matrix. Homogenates were centrifuged at $13,000 \mathrm{rpm}$ for $2 \mathrm{~min}$ at room temperature to remove gel debris and collect supernatants containing clean libraries. Afterwards, the libraries were re-amplified in 10 cycles of PCR amplification with QIAGEN Multiplex PCR Kit (Qiagen, Hilden, Germany), obtaining a volume of $60 \mu \mathrm{L}$ for each library. Finally, the cDNA constructs were purified and concentrated to $25 \mu \mathrm{L}$ final volume with the QIAquick PCR Purification Kit (Qiagen, Hilden, Germany). A brief work-flow chart figure to side by side compare the tradition method and the optimized Small RNA Library Preparation method was included (Fig. 1).

\section{Small RNA Library Preparation Workflow}

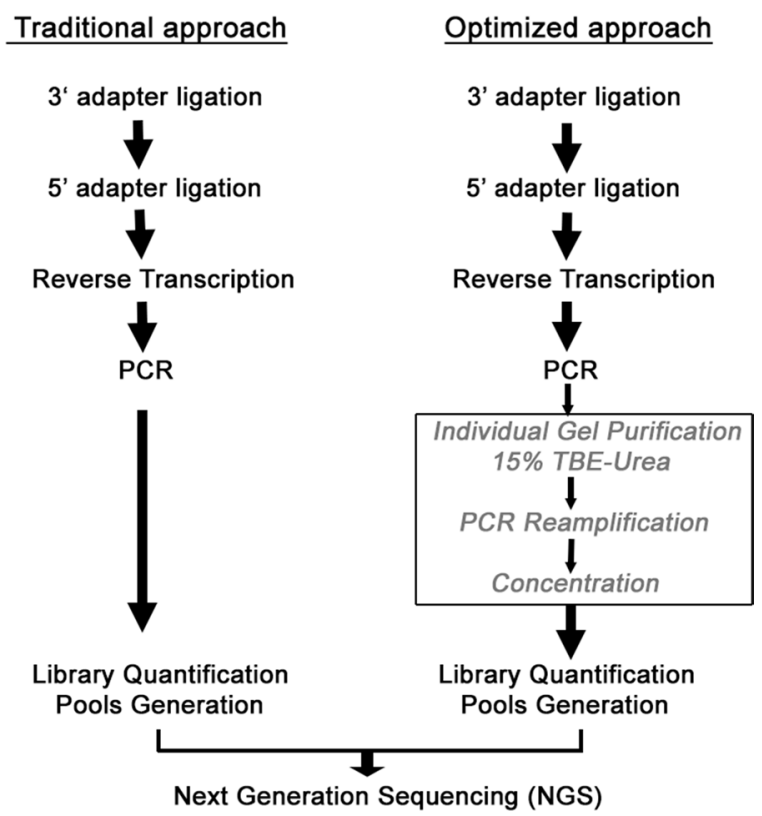

Fig. 1 A brief work-flow chart for optimized Small RNA Library Preparation. Comparison between the traditional method and our optimized Small RNA Library Preparation method from urinary exosomes, indicating the new additional steps (inside square) 


\section{Software, statistics and mapping}

To assess overall NGS data quality, adaptor sequences were trimmed from the $3^{\prime}$ end using Cutadapt (v.1.2.1), and reads without detectable adaptors were excluded from the data set. Sequence length distribution and base call accuracy were calculated with FastQC high throughput sequence data quality control software (Babraham Bioinformatics, UK, Version 0.11.4). This checking was performed before and after read cleaning. Trimmed reads were selected within desired ranges (18-26 bp and 24-33 bp) and aligned with Bowtie 2 v.0.12.7 [19], against the most recent human reference genome (GRCh38). Next, aligned reads were analyzed using HTSeq [20] against different databases to count how many reads mapped to each feature. In this study, miRNA features were obtained from the miRBase Sequence Database (Release 21) [21], piwiRNA features from the piRNA Bank (2018-12-09) [22], and other non-coding RNA features from NONCODE (2018) [23]. Statistical analyses were completed using GraphPad Prism software (GraphPad Software, Inc. La Jolla, CA, USA). Paired two-tailed Student's T test at significance level of $p<0.05$ was used to compare the effect of size selection on the small RNA mapped reads and miRNA sequencing, between purified and non-purified samples.

\section{Results \\ Modification of small RNA protocol does not suppress adapter-dimer formation}

The small RNA library prep kit allowed us to obtain the corresponding libraries for next-generation sequencing from urinary exosomes. However, the modified adapters used did not prevent adapter-dimer formation from exosome samples (Fig. 2). Electropherograms obtained for DNA Fragment Analysis by Capillary Electrophoresis showed a peak at 110-120 bp that corresponds to the amount of adapter-dimer (black box) and other peaks at 135-155 bp that correspond to small RNA types, miRNA and other sRNA (grey box). The high peak of sRNA libraries was accompanied by high levels of adapterprimer (Fig. 2a), and a low amount of sRNA was also accompanied by low or high adapter-dimer formation (Fig. 2b, c, respectively).

After CleanTag protocol optimization with an additional size selection step, we observed adapter-dimer formation in the gel before band extraction in non-purified (NP) samples 1 and 2, but no presence in purified (P) samples 1 and 2 (Fig. 3a). As electropherograms showed, adapter-dimer (black box) was totally removed after gel purification, and the sRNA library was enriched in the exosomal sRNA reads (Fig. 3b).

\section{Comparison of sequencing data including the size selection by gel within the CleanTag workflow}

When analyzing these two workflows with and without using a gel purification step, the total raw reads were statistically comparable, with slightly increase in non-purified samples (1.3\%) (Fig. 4a). However, sRNA mapped reads were higher in gel-purified samples with a $41 \%$ increase compared to non-purified ones $(p=0.007)$ (Fig. 4b), representing 33\% of total raw reads compared to the $20 \%$ in non-purified samples $(\mathrm{p}=0.009)$.

In addition, when we compared the effect of gel purification on miRNA mapped reads alone, we found that purified samples had a significant $37 \%$ increase compared to non-purified ones $(\mathrm{p}=0.008)$, and they represented $33 \%$ of the total raw reads compared to $19 \%$ of non-purified ones $(p=0.011)$ (Fig. 4c). From the other sRNA group analyzed, we obtained a two-fold increase in mapped reads between purified and non-purified samples ( $\mathrm{p}=0.027)$ (Fig. 4d). There was enrichment in sRNA reads, overall in miRNA data.

\section{Effect of size selection of PCR products by gel purification on tagged small RNA library population}

In order to determine whether the size selection step produced any meaningful changes in miRNA detection, we compared sequencing results with or without gel purification within our optimized workflow. We found a similar tagged miRNA population with or without the size selection step, obtaining a determinant coefficient of $\mathrm{R}^{2}=0.96, \mathrm{p}<0.0001$ after $\log ^{2}$ transformation (Fig. 5a). Furthermore, comparison of the two conditions with a Venn diagram showed that the purification protocol tagged more specific miRNA than the one without purification, although the majority (581 miRNA) of the tagged miRNA population were similar in the two conditions (Fig. 5b).

Finally, we analyzed the effect of gel purification on the other non-coding RNA (ncRNA) types. As with the miRNA population, the other ncRNA did not change in distribution, obtaining similar percentage between ncRNA types (Fig. 6a, b). In both purified and nonpurified conditions, Y-RNA were the most representative ( $66 \%$ and $54 \%$, respectively), followed by lncRNA (20\% and $26 \%$, respectively) and tRNA (5\% and $8 \%$, respectively).

\section{Discussion}

This study presents an optimized Small RNA library preparation workflow for NGS-based exosome-associated miRNA analysis. We completely eliminated adapterdimer formation and the sRNA mapped read count was increased, overall for miRNAs, without changing tagged miRNA population. 


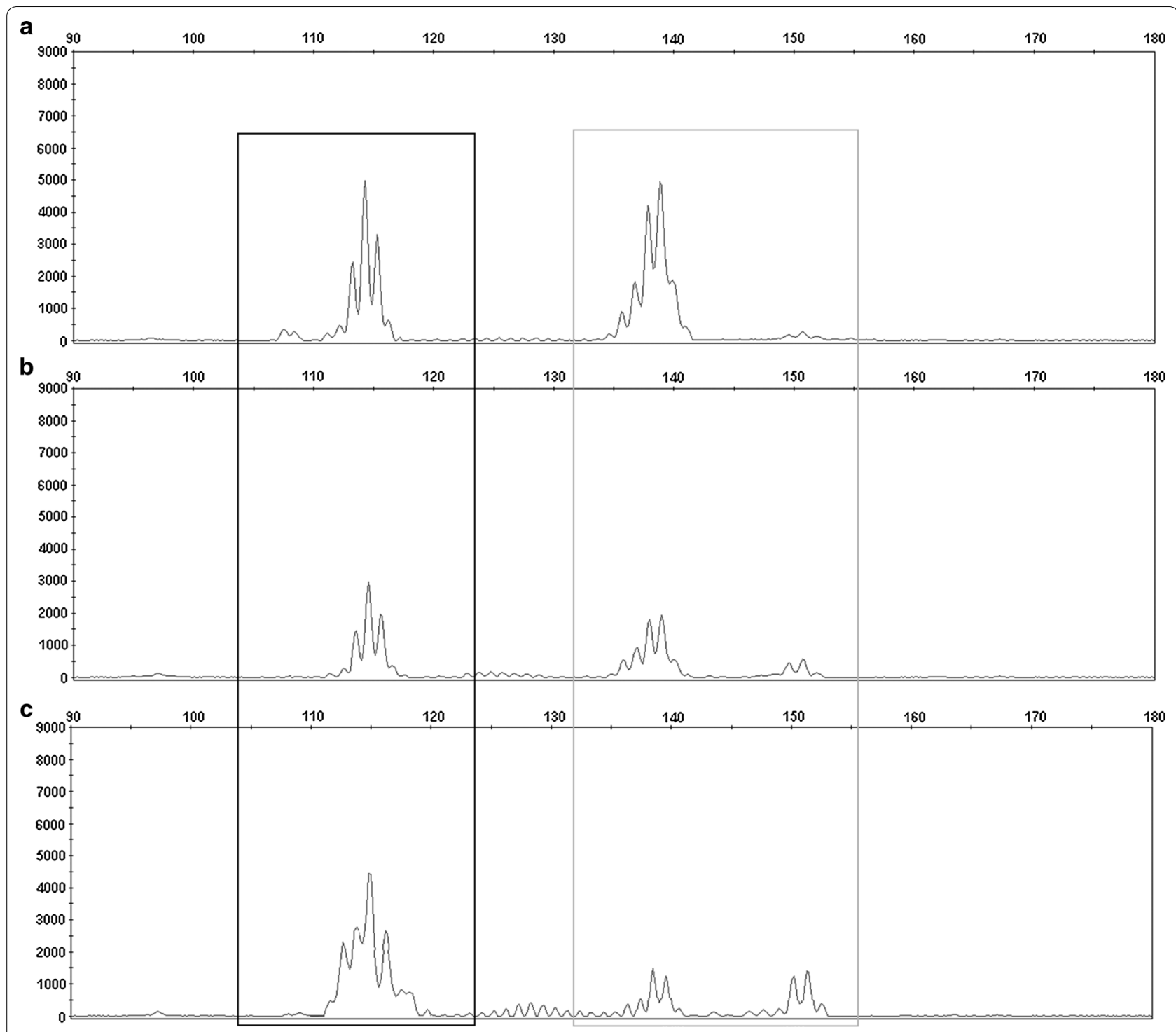

Fig. 2 Electropherograms obtained for DNA fragment analysis by capillary electrophoresis to analyze adapter-dimer formation. Three different cDNA libraries from urinary exosomal samples showed the presence of adapter-dimer (black box), high amount of adapter-dimer with high cDNA library (grey box) (a), low amount of adapter-dimer with low cDNA library (b), and high amount of adapter-dimer with low cDNA library (c)

The low quantity of RNA present in exosomes is a limiting factor for researchers. In response to this, a variety of small RNA sample preparation kits have implemented several modifications in their workflows to avoid adapterdimer formation and increase sRNA enrichment in samples with low RNA template input. As an example, to optimize sequencing performance the CleanTag Small RNA library prep kit builds libraries using specific adaptors designed for small amounts of starting material, suppressing adapter-dimer formation and eliminating the need for gel purification [17]. TruSeq ${ }^{\circledR}$ Small RNA Sample Prep Kit and NEBNext Multiplex Small RNA Library
Prep Set always implemented PAGE gel size-selection $[24,25]$. Many recent clinical studies have utilized these kits to analyze differential miRNA expression in exosome samples [26, 27], hence the vital importance of analyzing the potential effect of these modifications on miRNA data obtained by NGS from urinary exosomes.

Our results showed that libraries from urinary exosomes could be prepared using CleanTag Small RNA library prep, although it failed to prevent adapter-dimer formation entirely. We obtained high cDNA libraries with a high amount of adapter dimer and low cDNA libraries with high or similar adapter-dimer. This fact indicates 


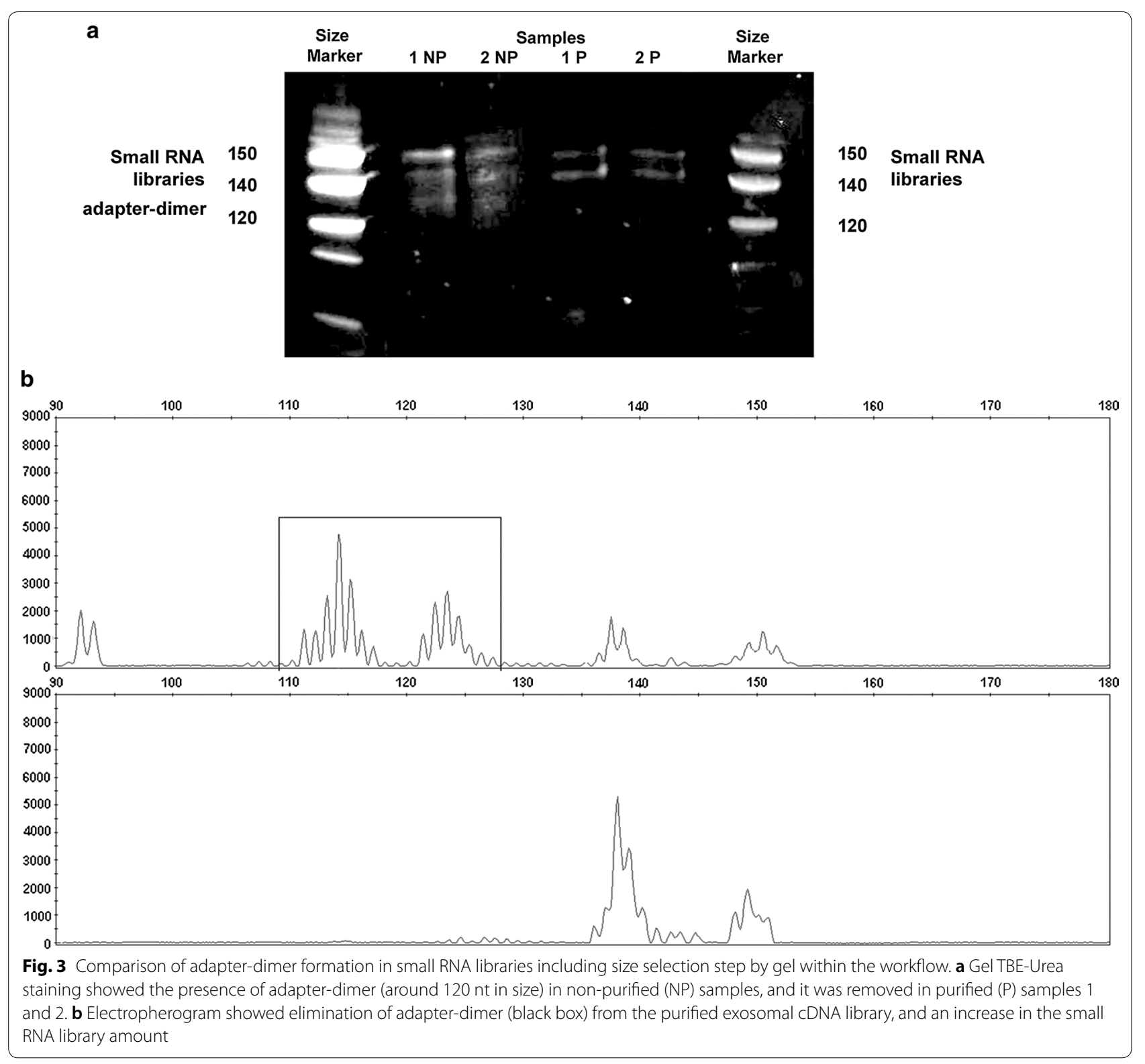

the need for a size selection step to purify the libraries for sRNA sequencing. When we analyzed the effect of gel purification on sRNA reads, we were able to demonstrate that including size selection within workflow efficiently tags sRNA and produces significantly more valuable reads and adapter dimer reduction compared to the protocol without gel purification. The robustness of our study lies in the analysis of results obtained from 24 individual cDNA libraries from urinary exosomes, which are more specific and sensitive for testing changes than working with library pools.

Human exosomes samples contain low abundance of sRNAs, and a unique or enriched set of miRNA compared to other EV subpopulations and cells [28, 29]. Identifying an exosomal miRNA signature associated with a pathological cellular state or disease has become increasingly important in recent years $[15,16]$. In this regard, to develop a protocol for enrich miRNA mapped reads from exosome samples. Our improved protocol showed not only an increase in miRNA mapped reads, but also no changes in the tagged miRNA population. Though there are slight differences between the two conditions, overall among miRNAs that had very low reads, these results provide evidence that within our workflow the size selection step itself produces an unbiased tagged miRNA population. 

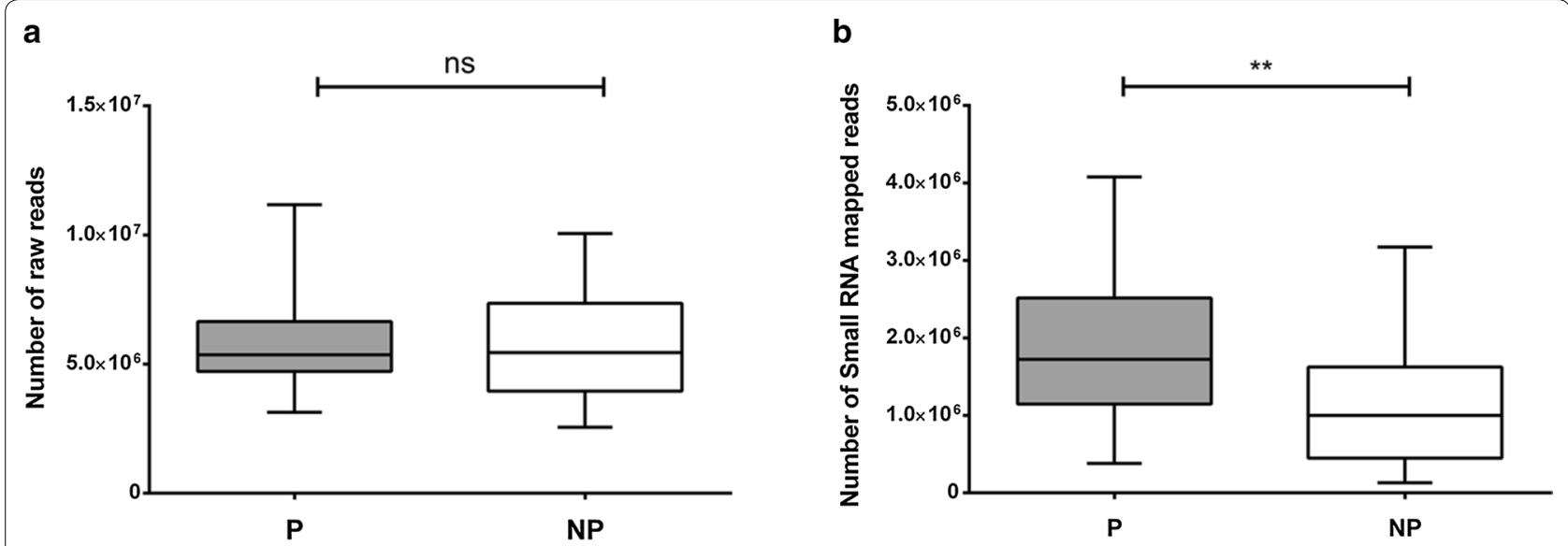

C

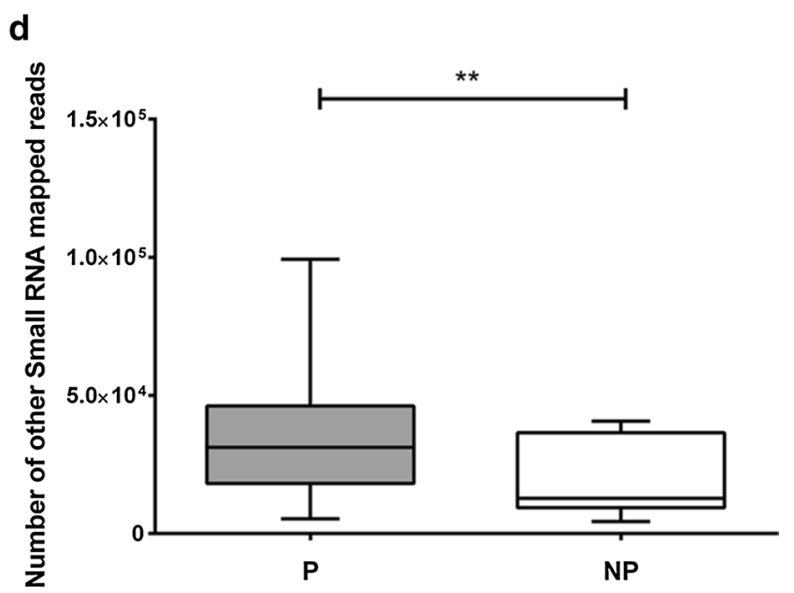

Fig. 4 Comparison of sequencing data including size selection step by gel within the workflow. Sequencing reads obtained from 24 urinary exosomal samples in each condition, purified (P) and non-purified (NP) condition. Analysis of total raw reads (a), small RNA mapped reads (b), miRNA mapped reads (c) and other small RNA mapped reads (d) between groups. Statistical significance: ${ }^{* *} \mathrm{p}<0.01$

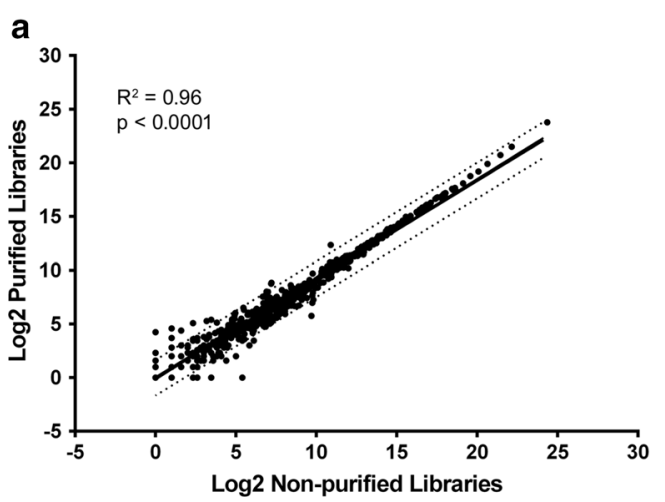

b

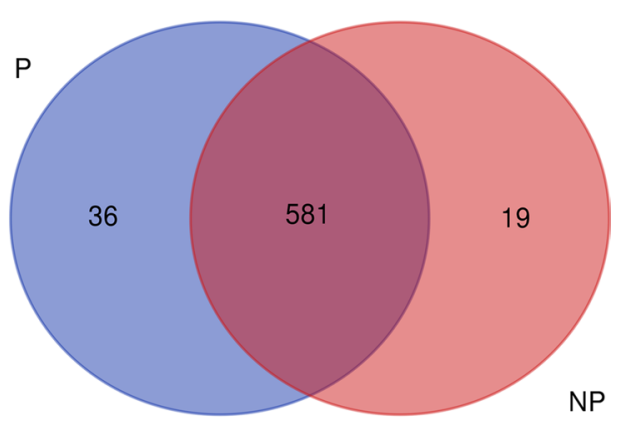

Fig. 5 Effect of size selection step on miRNA tagged library population. a Correlation plot of purified and non-purified libraries using the CleanTag library prep. Tagged miRNA are plotted after $\log ^{2}$ transformation. $\mathbf{b}$ Venn diagram of purified ( $P$ ) and non-purified (NP) libraries depicting number of urinary exosomal miRNA identified in all 24 samples for each condition 


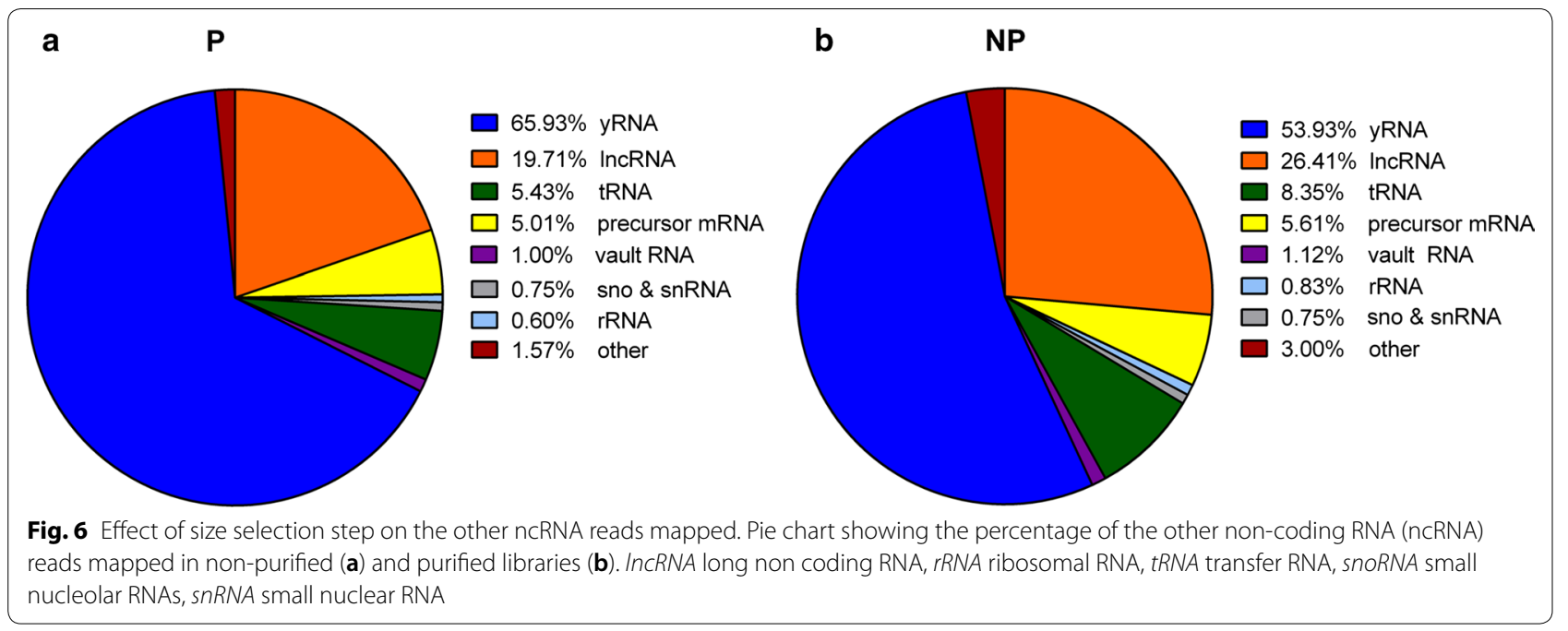

Furthermore, the number of mapped reads was augmented in the other sRNA species, without changes in the profile.

These findings prove that gel purification is specific for isolating the miRNA population from the urinary exosomes. This is because we were able to manually and carefully cut the band to between 145 and $160 \mathrm{nt}$, corresponding to miRNAs from the gel and avoid any other smaller or larger RNA populations in the samples. Further studies that analyzed exosome miRNA from other tissues it would be more universal. However, we think that whether this optimized protocol yields a higher amount of miRNA mapped reads without affecting the tagged miRNA population in urinary samples (biofluid with lower RNA input than plasma or plasma exosomes), could be applicable across other biofluids. We found purification by PAGE gel to be a very precise and specific method, particularly suitable for small sample size projects where miRNAs are the main focus.

\section{Conclusion}

Our results present an optimized NGS-based library preparation workflow for exosome-associated miRNA analysis. Firstly, adapter-dimer formation was totally eliminated from urinary exosome cDNA libraries. Secondly, this study provides evidence that within our small RNA library preparation workflow, the size selection step itself obtained high miRNA mapped reads from urinary exosomes without skewing the tagged miRNA population. Therefore, in order to prepare sRNA libraries for NGS-analysis from samples with low RNA input, it is important to carefully analyze the effect of their adapted library preparation workflow, to further validate the NGS-data obtained.

\section{Supplementary information}

Supplementary information accompanies this paper at https://doi. org/10.1186/s12967-020-02298-9.

Additional file 1: Table S1. Sequence Read Archive (SRA) and BioSample accession IDs of the samples analyzed.

\section{Abbreviations}

CDNA: Complementary DNA; miRNA: microRNA; ncRNA: Non-coding RNA; NGS: Next generation sequencing; PAGE: Polyacrylamide Gel Electrophoresis; sRNA: Small RNA; TBE: Tris-Borate-EDTA.

\section{Authors' contributions}

Conceived and designed the experiments: JPH, FJC, RC; performed the experiments: JPH, DO; Drafting the article: JPH, DO, RC; analyzed the data: JPH, DO, $D P G$; revising the manuscript: JR, RC; funding resources: JR, RC; providing intellectual content of critical importance to the work described: FJC, RC. All authors read and approved the final manuscript.

\section{Funding}

This study was supported by grants for research in health sciences of the Carlos III Health Institute (PI16/01402 and PI18/01405), and with ERDF funds.

\section{Availability of data and materials}

All data analysed during this study are included in this published article. The raw data are included in the BioProject PRJNA590749 and the corresponding Sequence Read Archive (SRA) and BioSample accession IDs are available in Additional file 1:Table S1.

\section{Ethics approval and consent to participate}

The study protocol was approved by the Ethics Committee of the Hospital Clínico Universitario of Valencia in accordance with the Declaration of Helsinki of 1975 as revised in 2008. All subjects have signed a written informed consent.

\section{Consent for publication \\ Not applicable.}

\section{Competing interests}

The authors declare that they have no competing interests.

\section{Author details}

${ }^{1}$ Cardiometabolic and Renal Risk Research Group, INCLIVA Biomedical Research Institute, Avd. Menéndez Pelayo, accesorio 4, 46010 Valencia, Spain. 2 Present Address: INSERM, U1016, Cochin Institute, 75014 Paris, France. 


\begin{abstract}
${ }^{3}$ Present Address: Genomics England, Dawson Hall, Charterhouse Square, London EC1M 6BQ, UK. ${ }^{4}$ Genomics and Diabetes Unit, INCLIVA Biomedical Research Institute, Avd. Menéndez Pelayo, accesorio 4, 46010 Valencia, Spain. ${ }^{5}$ CIBER of Diabetes and Associated Metabolic Diseases (CIBERDEM), Institute of Health Carlos III, Minister of Health, Barcelona, Spain. ${ }^{6}$ Internal Medicine, Clinic Universitary Hospital, Avd. Blasco Ibañez, 17, 46010 Valencia, Spain.
\end{abstract}

Received: 30 November 2019 Accepted: 11 March 2020

Published online: 18 March 2020

\section{References}

1. Berezikov E. Evolution of microRNA diversity and regulation in animals. Nat Rev Genet. 2011;12:846-60.

2. Kinet V, Halkein J, Dirkx E, Windt LJ. Cardiovascular extracellular microRNAs: emerging diagnostic markers and mechanisms of cell-to-cell RNA communication. Front Genet. 2013;4:214.

3. Calin GA, Croce CM. MicroRNA signatures in human cancers. Nat Rev Cancer. 2006;6:857-66.

4. Alevizos I, Illei GG. MicroRNAs as biomarkers in rheumatic diseases. Nat Rev Rheumatol. 2010;6:391-8.

5. Spornraft M, Kirchner B, Haase B, Benes V, Pfaffl MW, Riedmaier I. Optimization of extraction of circulating RNAs from plasma-enabling small RNA sequencing. PLOS ONE. 2014;9:e107259.

6. Kappel A, Keller A. miRNA assays in the clinical laboratory: workflow, detection technologies and automation aspects. Clin Chem Lab Med. 2017;55:636-47

7. Channavajjhala SK, Rossato M, Morandini F, Castagna A, Pizzolo F, Bazzoni $F$, et al. Optimizing the purification and analysis of miRNAs from urinary exosomes. Clin Chem Lab Med. 2014:52:345-54.

8. Perez-Hernandez J, Forner MJ, Pinto C, Chaves FJ, Cortes R, Redon J. Increased urinary exosomal microRNAs in patients with systemic lupus erythematosus. PLoS ONE. 2015;10:e0138618.

9. Perez-Hernandez J, Olivares D, Forner MJ, et al. Urinary exosome miR-146a is a potential marker of albuminuria in essential hypertension. J Transl Med. 2018;16:228.

10. Mathivanan $\mathrm{S}$, Ji H, Simpson RJ. Exosomes: extracellular organelles important in intercellular communication. J Proteom. 2010;73:1907-20.

11. Yáñez-Mó M, Siljander PR, Andreu Z, et al. Biological properties of extracellular vesicles and their physiological functions. J Extracell Vesicles. 2015;4:27066.

12. Valadi $H$, Ekström $K$, Bossios A, et al. Exosome-mediated transfer of mRNAs and microRNAs is a novel mechanism of genetic exchange between cells. Nat Cell Biol. 2007;9:654-9.

13. Pomatto MAC, Gai C, Bussolati B, Camussi G. Extracellular vesicles in renal pathophysiology. Front Mol Biosci. 2017;4:37.

14. Takahashi RU, Prieto-Vila M, Hironaka A, Ochiya T. The role of extracellular vesicle microRNAs in cancer biology. Clin Chem Lab Med. 2017;55:648-56
15. Greening DW, Gopal SK, Xu R, Simpson RJ, Chen W. Exosomes and their roles in immune regulation and cancer. Semin Cell Dev Biol. 2015:40:72-81.

16. Aghabozorgi AS, Ahangari N, Eftekhaari TE, et al. Circulating exosomal miRNAs in cardiovascular disease? Pathogenesis: new emerging hopes. J Cell Physiol. 2019;234:21796-809.

17. Shore S, Henderson JM, Lebedev A, et al. Small RNA library preparation method for next-generation sequencing using chemical modifications to prevent adapter dimer formation. PLoS ONE. 2016;11:e0167009.

18. Belair CD, Hu T, Chu B, Freimer JW, Cooperberg MR, Blelloch RH. Highthroughput, efficient, and unbiased capture of small RNAs from lowinput samples for sequencing. Sci Rep. 2019;9:2262.

19. Langmead B, Salzberg S. Fast gapped-read alignment with Bowtie 2. Nat Methods. 2012:9:357-9.

20. Anders S, Pyl PT, Huber W. HTSeq-a python framework to work with high-throughput sequencing data. Bioinformatics. 2015;31:166-9.

21. Kozomara A, Griffiths-Jones S. miRBase: annotating high confidence microRNAs using deep sequencing data. Nucleic Acids Res. 2014;42:D68-73.

22. Lakshmi SS, Agrawal S. piRNABank: a web resource on classified and clustered Piwi-interacting RNAs. Nucleic Acids Res. 2008;36:D173-7.

23. Zhao Y, Li H, Fang S, et al. NONCODE2016: an interactive database that aims to present the most complete collection and annotation of noncoding RNAs, especially long noncoding RNAs (IncRNAs). Nucleic Acids Res. 2016:44:D203-8.

24. Kuhlmann JD, Chebouti I, Kimmig R, et al. Extracellular vesicle-associated miRNAs in ovarian cancer-design of an integrated NGS-based workflow for the identification of blood-based biomarkers for platinum-resistance. Clin Chem Lab Med. 2019:57:1053-62.

25. Lopez JP, Diallo A, Cruceanu C, et al. Biomarker discovery: quantification of microRNAs and other small non-coding RNAs using next generation sequencing. BMC Med Genom. 2015;8:35.

26. Rodriguez M, Bajo-Santos $C$, Hessvik NP, et al. Identification of non-invasive miRNAs biomarkers for prostate cancer by deep sequencing analysis of urinary exosomes. Mol Cancer. 2017;16:156.

27. Min QH, Chen XM, Zou YQ, et al. Differential expression of urinary exosomal microRNAs in IgA nephropathy. J Clin Lab Anal. 2018;32:e22226.

28. Lunavat TR, Cheng L, Kim DK, et al. Small RNA deep sequencing discriminates subsets of extracellular vesicles released by melanoma cells_-evidence of unique microRNA cargos. RNA Biol. 2015;12:810-23.

29. Cheng L, Sharples RA, Scicluna BJ, Hill AF. Exosomes provide a protective and enriched source of miRNA for biomarker profiling compared to intracellular and cell-free blood. J Extracell Vesicles. 2014;26:3.

\section{Publisher's Note}

Springer Nature remains neutral with regard to jurisdictional claims in published maps and institutional affiliations.
Ready to submit your research? Choose BMC and benefit from:

- fast, convenient online submission

- thorough peer review by experienced researchers in your field

- rapid publication on acceptance

- support for research data, including large and complex data types

- gold Open Access which fosters wider collaboration and increased citations

- maximum visibility for your research: over 100M website views per year

At BMC, research is always in progress.

Learn more biomedcentral.com/submissions 